\title{
Therapeutic potential of heme oxygenase-1 in cardiovascular disease
}

\author{
Agnieszka JazWA*, Urszula FlorczyK, Jacek Stepniewski, Alicja Jozkowicz, Jozef Dulak* \\ Department of Medical Biotechnology, Jagiellonian University in Kraków, Poland \\ *Corresponding authors: jozef.dulak@uj.edu.pl and agnieszka.jazwa@uj.edu.pl
}

\begin{abstract}
Heme oxygenase-1 (HO1) degrades heme to carbon monoxide (CO), biliverdin, and ferrous iron. Through these products, HO1 mitigates cellular injury by exerting anti-oxidant, anti-apoptotic, and anti-inflammatory effects. Several lines of evidence indicate that angiogenic factors, such as vascular endothelial growth factor A (VEGF) and stromal cell-derived factor 1 (SDF1), mediate their proangiogenic action in endothelial cells and endothelial progenitor cells through induction of HO1, and reciprocally, VEGF and SDF1 are enhanced by $\mathrm{HO} 1$ overexpression. Ferrous iron released during the breakdown of free heme by $\mathrm{HO} 1$ is an extremely pro-oxidative molecule that can be rapidly removed by ferritin. Of note, this iron sequestering protein also has been shown to exert some proangiogenic effects. Moreover, our recent data indicate that HO1 is an important mediator of differentiation and function of stem cells, including endothelial and myoblasts progenitors. All of this makes HO1 a promising target for novel cardiovascular therapies. The aim of this review is to discuss the existing knowledge and to propose the therapeutic approaches, which have to consider the necessity of tight regulation of $\mathrm{HO1}$ expression.
\end{abstract}

Key words: angiogenesis, cytoprotection, heme oxygenase 1, iron, hypoxia

\section{Introduction}

The treatment of cardiovascular disorders such as coronary artery disease or peripheral artery disease, which are directly dependent on inadequate blood flow and insufficient oxygen content in tissues (hypoxia), still remains a challenging and difficult entity to manage with current surgical and standard medical procedures. Percutaneous transluminal coronary angioplasty, a life-saving medical procedure for acute myocardial infarction has its limitations, such as reocclusion and late restenosis. Sudden occlusion of a major coronary artery and acute myocardial ischemia causes rapid death of cardiomyocytes and vascular cells in the ischemic area (Olivetti et al., 1996). Reduced oxygen content from blood creates a condition in which the restoration of circulation results in inflammation and oxidative damage from reactive oxygen species (ROS). In fact, oxidative stress plays a major role in the pathophysiology of cardiac disorders. Upregulation of stress-inducible antioxidant proteins such as manganese superoxide dismutase, peroxisomal catalase, glutathione peroxidase- 1 and heme oxygenase 1 (HMOX1, HO1) constitutes a defense system for the heart enabling it to cope with an ischemic episo- de [reviewed in: (Jazwa et al., 2007)]. Insufficient and diminished with age antioxidant activity of these enzymes leads to inefficient defenses and worsening of symptoms. It is noteworthy that impairment of these protective reactions can be restored by gene transfer.

Gene therapy involves the insertion of a functional gene into cells to correct a cellular dysfunction or to provide a new cellular function. Recently, this procedure has been successfully used in the treatment of different monogenic diseases such as severe combined immunodeficiency syndrome (SCID) (Aiuti et al., 2009; Cavazzana-Calvo et al., 2000; Hacein-Bey-Abina et al., 2002), $\beta$-thalassemia (Cavazzana-Calvo et al., 2010; Roselli et al., 2010), adrenoleukodystrophy (Cartier et al., 2009) and Leber's congenital amaurosis (Maguire et al., 2009). The last two decades have also led to a significant progress in our understanding of the pathophysiology of heart failure at the molecular and cellular level, which created new possibilities of interventions available for gene therapy [reviewed in: (Rissanen and Yla-Herttuala, 2007)].

Several years of preclinical experimentation demonstrated that cardioprotection can be conferred by many different agents able to restore the function of failing 
cardiomyocytes. However, far from being successful in humans, this method still needs to be optimized by the choice of the most efficient transgene, proper route of administration, dose and gene carrier (Yla-Herttuala et al., 2004).

Myocardial ischemia triggers a spontaneous angiogenic response which is a growth of new blood vessels from the preexisting ones. Among many different agents precisely regulating this process, vascular endothelial growth factor A (VEGFA, VEGF) has been shown to play a pivotal role in its initiation. This protective angiogenic response is, however, usually not sufficient (Sasayama and Fujita, 1992). Importantly, angiogenesis can be stimulated in vivo and therefore represents an excellent therapeutic target for the treatment of cardiovascular disease.

VEGF, as already mentioned, is a critical regulator of angiogenesis that stimulates proliferation, migration, and proteolytic activity of endothelial cells (Ferrara, 1999), and is undeniably one of the well-studied factors for therapeutic angiogenesis for limb and cardiac repair [reviewed in: (Jazwa et al., 2007; Yla-Herttuala and Alitalo, 2003)]. VEGF expression is regulated by a variety of different stimuli among which the most potent inducer is hypoxia (Neufeld et al., 1999). We have previously shown that $\mathrm{HO} 1$ also upregulates the expression of $V E G F$ (Dulak et al., 2002; Jazwa et al., 2006) and is necessary for proper function of stromal cell-derived factor 1 (CXCL12, SDF1) (Deshane et al., 2007), the cytokine responsible for homing of endothelial progenitors and activation of mature endothelial cells. In fact, we discovered that $\mathrm{HO} 1$ functions both upstream and downstream of VEGF and SDF1 (Grochot-Przeczek et al., 2010), inducing their expression and being required for the proper response of endothelial or endothelial progenitor cells to VEGF or SDF1 stimulation (Fig. 1). Thus, both the direct and indirect protective and stimulatory effects on the vascular bed make $\mathrm{HO} 1$ a promising target for the improvement of treatment techniques in cardiovascular disorders.

\section{Heme oxygenase and its isoforms}

Heme oxygenases, $\mathrm{HO} 1$ and $\mathrm{HO} 2$, are two enzymes that catalyze the first, rate-limiting step in degradation of heme (Maines, 1997). Both isoforms cleave the $\alpha$ meso carbon bridge of heme, yielding equimolar quantities of carbon monoxide, ferrous iron $\left(\mathrm{Fe}^{2+}\right)$ and bili- verdin (Tenhunen et al., 1968, Maines et al., 1986). The breakdown of heme to these smaller compounds has its own significance in essential cellular metabolism - carbon monoxide has anti-inflammatory properties, biliverdin and free iron bound to a heavy chain ferritin (H-ferritin) are very efficient antioxidant molecules. These three enzymatic products, together with bilirubin resulting from conversion of biliverdin by biliverdin reductase (BVR), play a critical role counterbalancing oxidative stress and inflammation (Loboda et al., 2008; Maines, 1988). All together, these molecules have cytoprotective, proangiogenic and anti-apoptotic properties and are claimed to be the second, after glutathione, most important part of anti-oxidative protection system [reviewed in: (Dulak and Jozkowicz, 2003)].

$\mathrm{HO} 1$ is a $32-\mathrm{kDa}$ microsomal protein considered to be a protective, early stress-response agent that may have additional non-enzymatic activities related to its mitochondrial localization (Slebos et al., 2007) and nuclear translocation (Lin et al., 2007). The expression of $H O 1$ is generally very low in normal tissues, apart from liver and spleen, where it participates in the processing of senescent or damaged erythrocytes and in protection against oxidative damage caused by free porphyrins (Tenhunen et al., 1968). In all tissues, low basal expression of $\mathrm{HO} 1$ can be strongly and quickly upregulated by a wide variety of stimuli that cause oxidative stress, including its substrate heme, heavy metals, cytokines, ultraviolet rays, lipopolysaccharide, hydrogen peroxide, growth factors, nitric oxide and also by carbon monoxide (CO) [reviewed in: (Ryter et al., 2006)].

$\mathrm{HO} 2$ is a $36-\mathrm{kDa}$ protein which, in contrast to $\mathrm{HO} 1$, is constitutively and highly expressed in brain and testes (Rotenberg and Maines, 1990). Unlike HO1, HO2 does not seem to play any important role in conferring cytoprotection against oxidative stress. However, this second $\mathrm{HO}$ isoform has been shown to directly interact with the large conductance calcium-activated $\mathrm{K}+(\mathrm{BK})$ channels, and this complex has been suggested to play an important role in carotid body oxygen sensing (Williams et al., 2004).

\section{Intriguing biological functions of BVR}

HO1 activity is directly associated with BVR. Although the latter one was, for a long time, considered the sole enzyme that converts biliverdin to bilirubin, recent years have revealed unique features of this protein, 


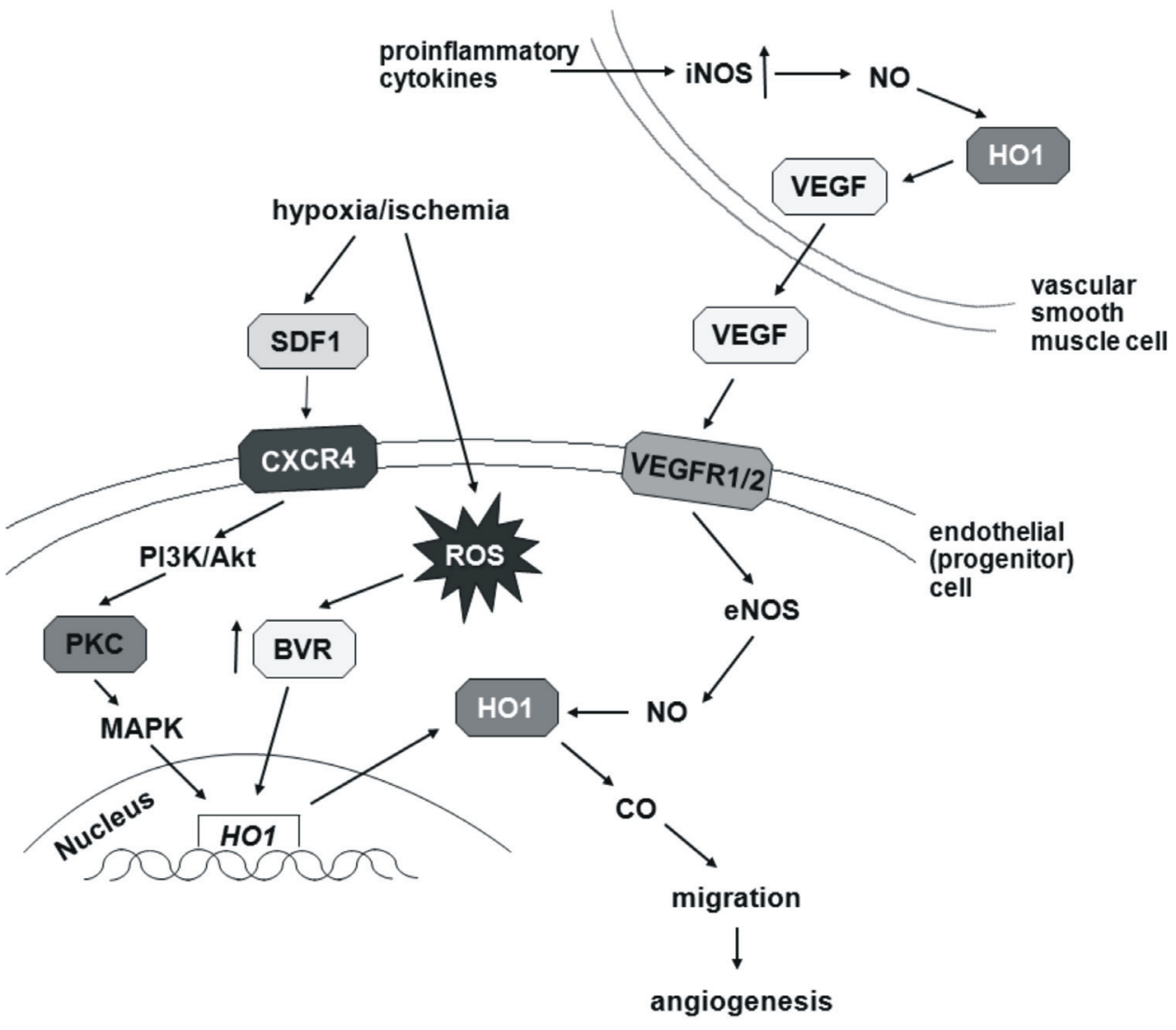

Fig. 1. Schematic model for HO1 signalling pathways and their effects on angiogenesis. Chemokine SDF1 is released at the site of injury (e.g., during tissue ischemia) and induces HO1 in endothelial (progenitor) cell. CO, one of the products of heme degradation, promotes migration of these cells and vascular repair. Pro-inflammatory cytokines acting on vascular smooth muscle cell cause iNOS up-regulation, which further leads to increased NO synthesis and HO1-mediated production of VEGF. VEGF acts on endothelial (progenitor) cells through VEGF receptor 1 (VEGFR1) or VEGF receptor 2 (VEGFR2). The proangiogenic effects of this agent involve, among others, HO1 upregulation

which are not related to its reductase capacity. Possessing the activity of a serine/threonine kinase, BVR is involved in glucose metabolism, control of cell growth and apoptosis and accordingly may play a role in pathogenesis of many disorders like diabetes or cancer. On the other hand, being a leucin zipper-like DNA binding protein, it can act as a transcription factor for activator protein 1-regulated genes [reviewed in: (Florczyk et al., 2008)]. As such BVR might specifically bind to HO1 promoter and mediate enzyme upregulation in response to oxidative stress (Ahmad et al., 2002) - Figure 1. A tight link between HO1 and BVR has been confirmed in 293A kidney cells, where silencing of $B V R$ gene with siRNA attenuated arsenite-mediated induction of $\mathrm{HO} 1$ and increased cell apoptosis (Miralem et al., 2005). Very recently we have demonstrated that enhanced activity of BVR, obtained with a doxycycline-induced Tet-On gene expression system, increased the expression of $\mathrm{HO}$ and was protective for NIH3T3 fibroblasts kept under stressful conditions arising from anti-cancer drugs treatment, cisplatin and doxorubicin. The effect was most probably related to PKC $\alpha / \beta$ activity as its inhibition reversed BVR action (Florczyk et al., 2010a). So far, data concerning $B V R$ expression in cancer are limited. Nevertheless, increased levels of BVR have been detected in kidney cancer cells and acute myeloid leukemia [reviewed in: (Florczyk et al., 2008)]. If this is a typical feature of different tumors, the strategy based on inhibition of BVR could be considered as an anti-cancer therapy. On the other hand, gene transfer-mediated overexpression of $B V R$ in normal cells might provide protection against potential cardiotoxic effects of chemotherapeutics (Outomuro et al., 2007). In this sense, increased expression of $B V R$ might be of potential therapeutic significance in cardiac injuries. 


\section{Regulation of rodent $\mathrm{Hol}$ and human $\mathrm{HO1}$ gene}

HO1 has been originally identified in rats as heat shock protein 32 (HSP32) because its expression is induced under stress conditions via heat shock factors, which activate heat shock element (HSE) within Hol promoter (Muller et al., 1987). Further analyses of the human $\mathrm{HO} 1$ and mouse $\mathrm{Ho} 1$ gene revealed a multiplicity of cis-acting DNA response elements serving as potential binding sites for transcription factors activated by different stimuli, which explains the highly inducible nature of this gene in both species.

A common feature for many agents capable of inducing $\mathrm{HO} 1$ is their electrophilic chemistry and generation of reactive oxygen and/or nitrogen species, which causes the activation of the transcription factor nuclear factor erythroid 2-related factor 2 (NF-E2-related factor 2, NRF2) (Florczyk et al., 2010b) (Fig. 2A). NRF2 belongs to a subset of the bZip family members that share a Cap " $\mathrm{n}$ " Collar domain and is maintained in the cytoplasm through binding to the cytoskeletal-associated protein Kelch-like ECH-associated protein 1 (KEAP1). Under normal conditions, NRF2 is sequestered in cytoplasm by KEAPI. The situation changes under stressful conditions, when electrophiles and/or ROS cause dissociation of NRF2 from KEAP1. As a consequence, NRF2 translocates to the nucleus and binds to antioxidant response element (ARE) within the promoter region of cytoprotective genes involved in electrophile conjugation, excretion of xenobiotics, ROS scavenging and stabilization of cellular redox potential. In this way NRF2 also activates $\mathrm{HO}$. Free heme, the primary substrate for $\mathrm{HO1}$, can act through NRF2 and increases the mRNA levels and activity of this protein by several folds (Yoshida et al., 1988). Many different factors (dimers, both hetero and homodimers) can bind to ARE including JUN, FOS, CREB, ATF, MAF and the Cap'n'Collar/basic leucine Zipper CNC-bZIP subclasses of the basic-leucine zipper (b-ZIP) family of transcription factors. These include activator protein 1, JUNB and JUND, NF- $\mathrm{KB}$, upstream stimulatory factors (USF1 and USF2), hypoxia-inducible factor $1 \alpha$ (HIF $1 \alpha)$, sterol regulatory element binding protein (SREBP-1), peroxisome proliferator-activated receptorsgamma (PPAR- $\gamma$ ), and others [reviewed in: (Jazwa and Cuadrado, 2010; Loboda et al., 2008)].

Small MAF family proteins also interact with other bZip members to repress $\mathrm{HO}$. These are $\mathrm{BACH} 1$ and
BACH2 (Oyake et al., 1996) and the p65 isoform of NRF1 (Wang et al., 2007). BACH1 contains multiple copies of a cysteine-proline motif surrounding the bZip domain that binds heme, thus leading to a decrease in its DNA-binding activity (Ogawa et al., 2001). This hypoxiainducible heme-regulated transcriptional repressor has two functions that seem to be important for $\mathrm{HO} 1$ regulation. Firstly, similar to NRF2, it can bind MAF proteins and then acts via ARE of the $\mathrm{HO} 1$ promoter; but, in contrast to NRF2, it represses $\mathrm{HO} 1$ transcription (Sun et al., 2002). Secondly, BACH1 binds heme with a high affinity, and this $\mathrm{BACH} 1$-heme interaction prevents BACH1 binding to ARE (Ogawa et al., 2001); therefore, heme abrogates the repressor function of BACH1. Heme also has the ability to regulate the nuclear export of BACH1 (Suzuki et al., 2004).

HIF $1 \alpha$ is another important regulator of HO1 (Lee et al., 1997) - Figure 2B. HIF1 is a basic helix-loop-helix heterodimeric transcription factor that consists of two subunits: oxygen-sensitive (HIF1 $\alpha$ ) and oxygen-insensitive constitutively expressed (HIF $1 \beta$ ). HIF $1 \alpha$ and HIF $1 \beta$ heterodimer binds to the hypoxia responsive element (HRE) in the promoter region of various genes whose products like erythropoietin, VEGF and mouse Ho1, to mention a few, play an adaptive role under hypoxic conditions (Semenza, 2004). Among multiple HIF isoforms, HIF $1 \alpha$ and HIF $2 \alpha$ are the best characterized. HIF $2 \alpha$ has a similar structure to HIF $1 \alpha$, but in contrast to widely present HIF $1 \alpha$, the occurrence of HIF $2 \alpha$ is restricted to certain tissues such as heart, lungs, kidney and endothelium (Stachurska et al., 2010; Wiesener et al., 2003). At normal oxygen tension, the HIF $1 \alpha$ subunit is rapidly degraded. Degradation is mediated by a family of prolyl hydroxylases (PHD1, 2 and 3) that hydroxylate proline residues in the oxygen-dependent degradation domain of HIF1 $\alpha$ for recognition by the von Hippel-Lindau (VHL) complex that subsequently targets it for degradation via proteosome. PHDs need the presence of oxygen, iron, and 2-oxoglutarate (2-OG), and therefore their activity can be diminished by hypoxia, iron chelators, or 2-OG analogues such as dimethyloxaloylglycine (DMOG) [reviewed in: (Loboda et al., 2008)].

Apart from HSE, ARE, and HRE, many other positive regulatory elements have been found in the $\mathrm{HO}$ promoter, for e.g. cadmium-responsive element, SMADbinding element, consensus binding sites for AP 1 , speci- 


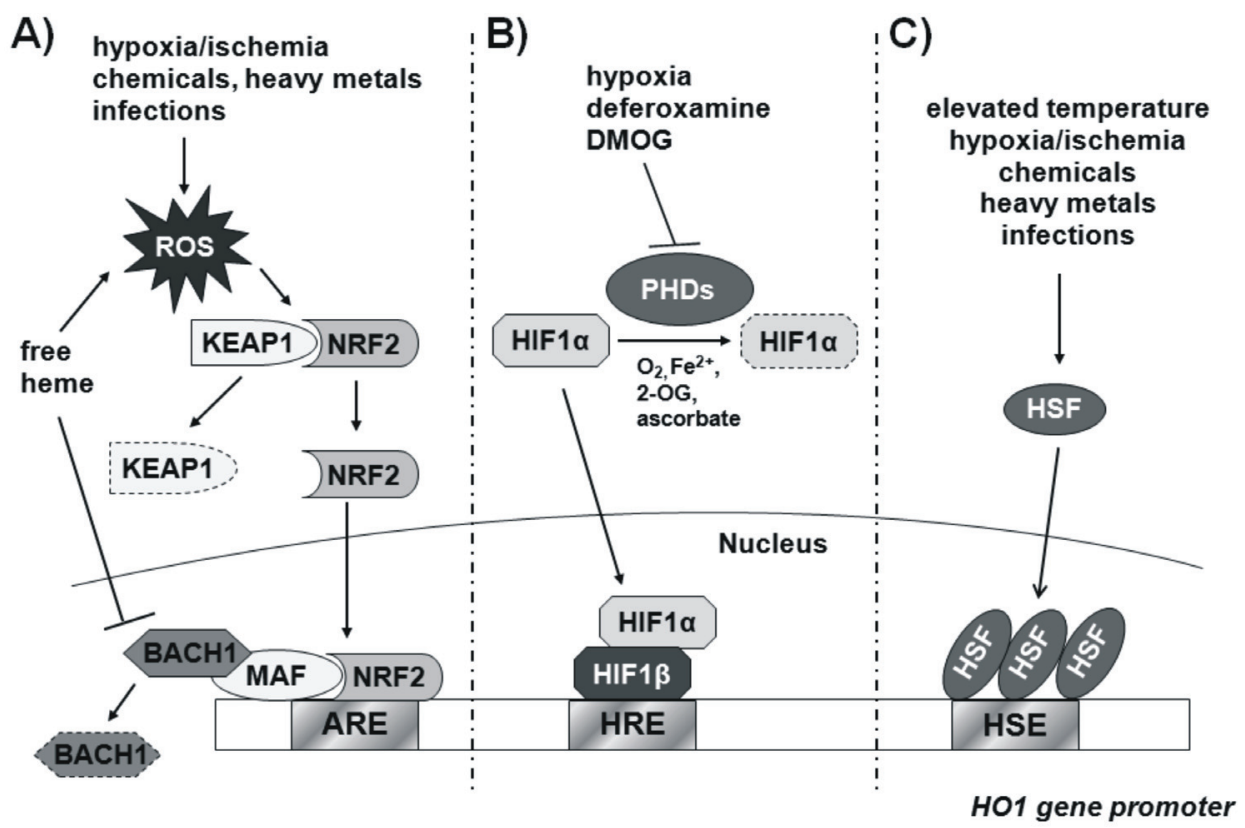

Fig. 2. Schematic model for HO1 gene regulation via NRF2, HIF1 $\alpha$ and HSF. A) HO1 can be induced by its substrate heme as well as by a variety of agents increasing intracellular levels of ROS. ROS induce ubiquitin-dependent proteasomal degradation of KEAP1 (dotted line) enabling the NRF2 translocation to the nucleus and, in the form of heterodimer with small MAF proteins, activation of antioxidant response element (ARE). BACH1 is a transcriptional repressor of the $H O 1$ gene in human cells. Under normal conditions, BACH1 is associated with small MAF proteins and limits the interaction between MAF and NRF2. Heme limits BACH1 expression and stabilizes NRF2, which can form heterodimers with MAF. In the presence of high levels of heme and oxidative stress, BACH1 is exported from the nucleus and degraded (dotted line), which allows derepression of the $\mathrm{HO} 1$ gene. B) Hypoxic regulation of $\mathrm{HO} 1$ involves activation of the hypoxia response element (HRE). In normoxia, HIF $1 \alpha$ is hydroxylated by proline hydroxylases (PHDs) in the presence of $\mathrm{O}_{2}, \mathrm{Fe}^{2+}, 2$-oxoglutarate (2-OG) and ascorbate. Hydroxylated $\mathrm{HIF} 1 \alpha(\mathrm{OH})$ is directed to ubiquitin-dependent proteaseomal degradation (dotted line). Under hypoxic conditions, iron chelators (deferoxamine) or 2-OG analogues (DMOG) proline hydroxylation is inhibited, which leads to HIF1 $\alpha$ accumulation and translocation to the nucleus. There, HIF $1 \alpha$ dimerises with HIF1 $\beta$ and binds to HRE. C) Cells exposed to elevated temperatures or other stress stimuli respond by an increased expression of heat shock proteins, such as HO1. The stress response is controlled primarily at the transcription level by a heat shock factor (HSF). In unstressed cells, HSF is present in the cytoplasm and the nucleus in a monomeric form that has no DNA binding activity. In response to stress, the monomeric HSFs combine into trimers and accumulate within the nucleus where they bind to heat shock element (HSE)

ficity protein 1 , nuclear factor-KB (NF- $\mathrm{kB}$ ) and activator protein 2, STATx, and upstream stimulatory factor [reviewed in: (Jazwa and Cuadrado, 2010; Loboda et al., 2008)].

\section{Polymorphisms in the human $\mathrm{HO} 1$ promoter}

To date, three polymorphisms in the 5 'flanking region of the human $\mathrm{HO} 1$ gene have been described: a GT dinucleotide length polymorphism, and two single nucleotide polymorphisms, $-1135 \mathrm{~A} / \mathrm{G}$ and $-413 \mathrm{~A} / \mathrm{T}$ (Exner et al., 2004). All of them can modulate the level of $\mathrm{HOI}$ transcription that has been associated with the incidence and/or progression of a variety of diseases.

The most common variation in $\mathrm{HO}$ expression levels in the human population appears to be due to dif- ferent lengths of the GT repeats. The GT repeat length ranges from 12 to 40 and usually shows a bimodal distribution, with the median length of the short allele being around 23 repeats and that of the long allele being around 30 dinucleotide GT repeats in different populations studied (Exner et al., 2004). This purine-pyrimidine-alternating sequence, possessing Z-conformation potential, can negatively affect transcription of $\mathrm{HO1}$ (Hill-Kapturczak et al., 2003). Longer GT sequences in this region have been associated with attenuated $\mathrm{HO}$ transcriptional activity and weaker response to oxidative stress (Hirai et al., 2003; Takahashi et al., 1999; Yamada et al., 2000).

Although the recently performed large scale analysis did not confirm a meaningful effect of $\mathrm{HO} 1$ promoter 
polymorphism on coronary artery disease or myocardial infarction (Lublinghoff et al., 2009), there are many clinical data indicating that individuals carrying fewer repeats and thus having stronger $\mathrm{HO}$ expression are less prone to certain cardiovascular pathologies. Thus, the presence of longer, less active alleles was associated with an increased risk of higher incidence of coronary artery disease in type 2 diabetic or hemodialyzed patients (Brydun et al., 2007; Chen et al., 2002), elevated rate of restenosis after balloon angioplasty (Gulesserian et al., 2005) or more frequent aortic aneurysms (Morgan et al., 2005) and ischemic cerebrovascular events (Funk et al., 2004). Moreover, among patients with peripheral artery disease, those carrying longer $\mathrm{HO} 1$ alleles had higher rates of myocardial infarction, percutaneous coronary interventions, and coronary bypass operations (Dick et al., 2005). We have recently demonstrated that primary human umbilical vein endothelial cells (HUVEC) carrying shorter $\mathrm{HO}$ alleles demonstrated better antioxidative status (Taha et al., 2010). These cells not only survived better under oxidative stress conditions, but also proliferated more effectively in response to VEGF, and produced less proinflammatory mediators (Taha et al., 2010). This indicates, for the first time, a significance of small and perhaps also diagnostically significant variations in $\mathrm{HOI}$ expression in vasculature.

\section{HO1 in the vascular system}

Angiogenesis, the process of creating new blood vessels from preexisting ones, is a key step in multiple physiologic and pathologic processes such as wound healing, menstruation cycle, tumor growth and metastasis (Ferrara, 1999). Under normal conditions, the process of angiogenesis is precisely regulated by a balance of multiple pro- and antiangiogenic factors. The impairment of pregnancy in animals lacking the functional $\mathrm{Hol}$ gene $\left(\mathrm{HoI}^{\prime}\right)$ and the significant mortality of $\mathrm{HoI}^{\prime-}$ embryos (Poss and Tonegawa, 1997) might suggest a meaningful role of $\mathrm{HO} 1$ in prenatal angiogenesis. In fact, several studies that demonstrated the importance of $\mathrm{HO} 1$ in the vascular system, including vascular tone regulation, anti-smooth muscle proliferation, antiendothelial apoptosis, and angiogenesis [reviewed in: (Dulak et al., 2008)] indicated that this protein may be important in postnatal angiogenesis.

The first study linking HO1 to the process of new blood vessels formation was published by Deramaudt and coworkers (1998) who showed that overexpression of $\mathrm{HO1}$ in endothelial cells enhances their proliferation. Additional experiments confirmed that $\mathrm{HO} 1$ promotes endothelial cell cycle progression with a reduction in p21 and p27 in endothelial cells (Li Volti et al., 2005). The relationship between HO1 and VEGF was carefully investigated. Several studies, including ours, demonstrated that this potent proangiogenic growth factor can activate HO1 in endothelial cells (Bussolati et al., 2004; Dulak et al., 2002; Jozkowicz et al., 2003b) - Figure 1. Moreover, inhibition of $\mathrm{HO} 1$ activity by tin protoporphyrin prevented hypoxia-induced VEGF synthesis in smooth muscle cells indicating the close interaction between both proteins (Dulak et al., 2002).

As mentioned above, VEGF initiates a complex process of angiogenesis. At the same time this protein, also known as vascular permeability factor, increases vascular permeability and allows plasma components and inflammatory cells to exit the bloodstream. Additionally, inflammatory cells can release various angiogenic cytokines including VEGF (Sunderkotter et al., 1994). When taking into account the anti-inflammatory properties of HO1, one might think about a dual effect of this enzyme in the vascular system. It seems that inflammation-induced angiogenesis, believed to play an important role in e.g. in tumor growth, might be attenuated by increasing HO1 activity in macrophages (Bussolati et al., 2004).

Hypoxic stimulation of gene expression is mediated by the transcription factor HIF $1 \alpha$ which binds to HRE in the promoter region of several target genes involved in the modulation of angiogenesis, ATP synthesis, oxygen supply, and cell survival. Over the past two decades more than 100 genes have been described as being regulated by HIF1 $\alpha$ (Pouyssegur et al., 2006). In fact, HRE sequence is also present in the $\mathrm{HO} 1$ promoter and $\mathrm{HO}$ expression has been shown to be induced by hypoxia in rodent vascular cells via HIF1 (Lee et al., 1997). Interestingly, however, in many human cells, particularly endothelial, the expression of $\mathrm{HO} 1$ is not induced or even downregulated under hypoxic conditions (Kitamuro et al., 2003). This is most probably associated with the increased levels of BACH1, a hypoxia-inducible repressor of $\mathrm{HO1}$ (Fig. 2A). Nevertheless, the stimulatory effect of hypoxia (1\% oxygen) on Vegf production by rat vascular smooth muscle cells can be reverted by inhibitors of Ho1 activity (Dulak et al., 2002). In human HaCaT keratinocytes, hypoxic induction of HIF1 upregu- 
lates $V E G F$ in an HO1-dependent manner, as the effect is blunted by inhibitors of $\mathrm{HO} 1$ activity or HO1 siRNA (Jazwa et al., 2006).

The proangiogenic effects of $\mathrm{HO}$ appear to be mediated in a large part by the actions of its reaction byproduct, $\mathrm{CO}$. CO, similar to nitric oxide, is able to activate soluble guanylate cyclase (Coceani, 1993). Once activated, this enzyme converts GTP to cGMP and increased intracellular cGMP leads to vasodilatation, inhibition of smooth muscle cell proliferation, anti-thrombogenic effects, and anti-inflammatory responses in vascular system (Forstermann and Munzel, 2006). Our studies have demonstrated that $\mathrm{CO}$ can promote angiogenesis via several mechanisms, including stimulation of $V E G F$ expression (Fig. 1). The treatment of vascular smooth muscle cells with 1\% CO gas (Dulak et al., 2002) or endothelial cells with CO-releasing molecule 2 (Jozkowicz et al., 2003b) induces VEGF production. Moreover, very recently $\mathrm{CO}$ has been shown to stimulate angiogenesis in a paracrine mode of action because CO-mediated increase in HIF $1 \alpha$ protein level in astrocytes induced secretion of VEGF and led to the subsequent activation of adjacent and surrounding endothelial cells to promote angiogenesis (Choi et al., 2010). Interestingly, the authors observed that CO increases HIF $1 \alpha$ level by two distinct mechanisms: upregulation of protein synthesis by activating the translational regulatory proteins p70 S6 kinase and eIF-4E through PI3K/AKT and MEK/ERK pathways and inhibition of HIF $1 \alpha$ degradation by promoting the interaction of HIF $1 \alpha$ with HSP90 $\alpha$ (Choi et al., 2010). The synergistic effect of HO1 and HIF $1 \alpha$ activities has recently been demonstrated in vivo. A combined delivery of their genes reduced apoptosis, increased VEGF secretion and improved angiogenesis in ischemic mouse hindlimbs to a greater extent than HO1 or HIF $1 \alpha$ single-gene therapy (Bhang et al., 2010).

In addition to VEGF, SDF1 has also been shown to play an important role in the postnatal angiogenesis. SDF1 is a chemokine that binds to a single high affinity receptor, CXCR4. The main role of SDF1 is to promote the migration of bone marrow-derived CXCR4+-endothelial progenitor cells as well as hematopoietic cells to local tissues, where they participate in neovascularization (Ceradini and Gurtner, 2005). SDF1 increases HO1 expression, which in turn mediates SDF1-induced angiogenic response of endothelial cells (Deshane et al., 2007) - Figure 1. HO1 deficiency in endothelial cells causes defective angiogenesis upon SDF1 stimulation. Recently, HO1 has been shown to promote endothelial progenitor cell mobilization and neovascularization after hindlimb ischemia in mice (Tongers et al., 2008). In line with these findings, local (Lin et al., 2008) and systemic (Lin et al., 2009) HO1 gene transfer via adeno-associated virus promoted neovascularization in the infarcted hearts through the concurrent induction of VEGF and SDF1 and the recruitment of bone marrow-derived c-kit+-stem cells. Concomitant administration of both VEGF and SDF1 neutralizing antibodies has significantly attenuated HO1-mediated neovascularization and protection during myocardial infarction, highlighting the cooperative roles of both factors in HO1-mediated angiogenesis and protection (Lin et al., 2008).

The cardioprotective effects of Hif1 $\alpha$ subunit have been shown to be dependent on HO1 activity (Czibik et al., 2009). Very recently it has been suggested that HO1 and CO may actually have differential roles in cardiac regeneration after myocardial infarction (Lakkisto et al., 2010). CO, delivered in a form of methylene chloride prior to coronary artery ligation in rats, promoted vasculogenesis and myocardial regeneration by activating c-kit+stem/progenitor cells and promoting the differentiation of stem cells to form new arteries and cardiomyocytes through induction of Hifla, Sdf1 $\alpha$ and vascular endothelial growth factor B synthesis. Instead, induction of endogenous $\mathrm{Ho} 1$ with cobalt protoporhyrin IX appeared to promote angiogenesis by a CO-independent mechanism as Ho1 induced Sdf1 $\alpha$ expression only (Lakkisto et al., 2010). Although the authors do not comment on that, it is possible that in their experimental setup, other Ho1 activity products were involved in cardiac regeneration and vascularization. It is also important to emphasize that such $\mathrm{HO} 1$ inducers like protoporphyrins may exert different unspecific effects (Jozkowicz and Dulak, 2003a) and CoPPIX has even been shown to downregulate the HIF1 $\alpha$ expression in adult T-cell leukaemia cells (Hamamura et al., 2007).

\section{Role of iron and ferritin in HO1-mediated neovascularization}

Heme released from a series of hemoproteins, including hemoglobin and cytochrome P450, is the natural substrate for HO1 that increases its mRNA and activity by several-fold (Yoshida et al., 1988). Once metabolized by $\mathrm{HO} 1$ it gives rise to $\mathrm{CO}$, biliverdin, and iron ions. Iron 
is an essential metal for all living organisms participating in cellular processes, such as DNA synthesis, enzyme functions, and oxygen transport. However, the biological use of iron is limited by its low solubility and its propensity to participate in the formation of potentially lethal oxidizing agents. In the presence of superoxide and hydrogen peroxide $\left(\mathrm{H}_{2} \mathrm{O}_{2}\right)$, ferrous iron $\left(\mathrm{Fe}^{2+}\right)$ can catalyze formation of the hydroxyl radical which is a powerful oxidizing agent promoting lipid peroxidation, mutagenesis, DNA strand breaks, activation of oncogenes, and tumor suppressor gene inhibition. Therefore, the release of high amounts of reactive iron under the conditions of strongly enhanced $\mathrm{HO}$ activity and a high supply of heme may be potentially harmful to the cells (Suttner and Dennery, 1999). On the other hand, HO1-dependent release of free iron during heme catabolism results in the upregulation of a secondary antioxidant protein, ferritin (Eisenstein et al., 1991).

Ferritin is an intracellular protein that stores iron in a nontoxic, yet bioavailable form (Torti and Torti, 2002). This protein also exists in serum but has distinctive subunit composition and low iron content. Ferritin is a binding partner of high molecular weight kininogen (HK), a plasma protein involved in the intrinsic coagulation cascade. Proteolytic cleavage of HK by kallikrein produces two molecules that actually have opposing roles in angiogenesis: proangiogenic bradykinin and 2-chain high molecular weight kininogen $(\mathrm{HKa})$ which is antiangiogenic. It has been demonstrated that through the binding to the antiangiogenic domain of $\mathrm{HKa}$, ferritin antagonizes HKa's effects, leading to increased blood vessel growth in a mouse tumor model (Coffman et al., 2009). Accordingly, we have recently shown that upregulation of ferritin in endothelial cells correlates with $\mathrm{HO} 1$ inducibility and is significantly augmented in HUVEC cells carrying cytoprotective short alleles of $\mathrm{HO} 1$ promoter (Taha et al., 2010).

Iron can indirectly influence angiogenesis by acting as a cofactor for enzymes involved in regulation of VEGF synthesis or by enhancing oxidative stress (Ponka, 1999). Interestingly, we have previously observed that deferoxamine, a nonpermeable iron chelator that extrudes iron from cells, significantly enhanced Vegf synthesis, whereas iron diminished its release by rat vascular smooth muscle cells, both effects being mediated by modulation of Hif1 activity (Dulak et al., 2002). Thus, it can be hypothesized that an increased iron content might, under certain conditions, attenuate VEGF-dependent angiogenesis. It seems that these effects may vary between cells and may result from their different capabilities to achieve cellular iron homeostasis by upregulation of HO1 or ferritin, or modulation of iron efflux. It has been demonstrated that the extrusion of iron from the cells requires active $\mathrm{HO} 1$ and it can be hypothesized that the effect of HO1 on VEGF synthesis can be related to the iron-pump activity of HO1 (Baranano et al., 2000; Ferris et al., 1999).

\section{Exogenous administration of $\mathrm{HO1}$ by gene therapy}

Several lines of evidence indicate an important role of $\mathrm{HO} 1$ in the cardiovascular system. The induction of HO1 by gene therapy inhibits atherogenic plaque formation (Juan et al., 2001), attenuates vascular remodeling following balloon injury (Tulis et al., 2001) and reduces the infarct size in rats subjected to myocardial infarction (Melo et al., 2002). These beneficial effects seem to be related to the anti-proliferative, anti-thrombotic and antiinflammatory action of the end products of heme degradation. Additionally, the therapeutic effects of HO1 in several preclinical models of cardiovascular disease have been linked to increased angiogenesis.

In a rat model of hindlimb ischemia, adenoviral delivery of rat $H o 1$ enhanced angiogenesis in the ischemic muscles through the production of endogenous Vegf, an effect being abrogated by inhibition of Hol activity (Suzuki et al., 2003). Importantly, it has been shown that excessive accumulation of reactive iron at high $\mathrm{HO} 1$ levels may lead to increased oxidative stress, cytotoxicity, and abnormal cellular proliferation (Suttner and Dennery, 1999). Therefore, when thinking about $\mathrm{HO1}$ gene transfer it is worth emphasizing the importance of a tight regulation of this gene expression. This objective can be achieved by different means.

The concept of hypoxia-regulated $\mathrm{HO} 1$ gene transfer was intensively investigated in different experimental studies (Pachori et al., 2004; Pachori et al., 2006; Tang et al., 2005; Tang et al., 2004) and seems to be a suitable strategy for the treatment of ischemic diseases by reducing cellular damage during ischemia-reperfusion injury, and preserving heart functions. This system is based on HIF $1 \alpha /$ HRE-mediated upregulation of the introduced gene (Fig. 3). The main advantage of the use of hypoxia-regulated vectors containing HRE sequence res- 


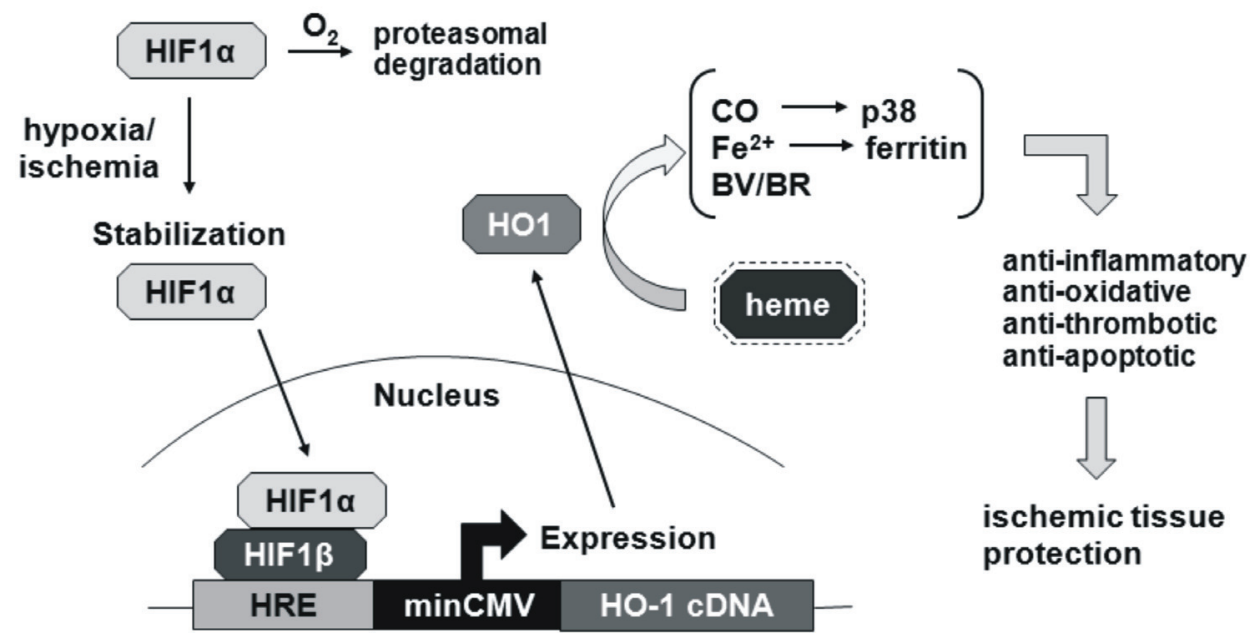

Fig. 3. The model of hypoxia-regulated $\mathrm{HO1}$ expression. cDNA coding for $\mathrm{HO} 1$ is located downstream of minCMV promoter and HRE sequence which constitutes the binding site for HIF $1 \alpha / \beta$ heterodimer that activates transcription only in the absence of oxygen, which happens e.g. under ischemic conditions associated with myocardial infarction. Increased HO1 activity generates $\mathrm{CO}$, which is a potent anti-inflammatory, anti-thrombotic and anti-apoptotic compound at low, physiological concentrations. Its effect is, to a large extent, dependent on the activation of p38 mitogen-activated protein kinase. Heme-derived iron triggers pathways involving the activation of ferritin, that further leads to its sequestration. Biliverdin (BV) and bilirubin (BR) produced from $\mathrm{HO} 1$ activity also contribute to the cellular anti-oxidant balance. Altogether, an increased HO1 activity can exert protective effects in ischemic tissues.

ponsible for sensing hypoxia is the induction of the transgene expression in hypoxic tissues without therapeutic protein production in tissues unintentionally transfected or transduced with a plasmid or viral vector. Moreover, unlike the constitutive promoters which lead to a constant production of proteins, the hypoxia-regulated promoters enable overexpression of a therapeutic agent that helps to overcome the ischemic assault in statu nascendi and creates the possibility of a tight regulation of $\mathrm{HO} 1$ expression (Fig. 3).

Another system providing control over the dosage and temporal expression of a given gene product is based on antibiotic-regulated vectors. The tetracycline (Tet) On/Off gene control system has the prokaryotic origin and is based on Tet resistance operon of $\mathrm{E}$. coli. Briefly, in the Tet-Off system the addition of an antibiotic results in inactivation of transcription, while using TetOn system, the presence of an antibiotic activates the expression of the gene of interest. Importantly, the TetOn system is about 100 times more sensitive to the Tet analogue, doxycycyline, and therefore doxycycyline is frequently used in this kind of studies. Tet-On/Off system was used in different experimental settings including those based on $\mathrm{HO} 1$ overexpression (Suttner and Dennery, 1999).

\section{Role of HO1 in stem and progenitor cell function and efficiency of cell therapy}

Cell therapy, similar to gene therapy, has been claimed to be effective in promoting neovascularization in various preclinical models. Bone marrow-derived stem/ progenitor cells differentiate into circulating endothelial progenitor cells (EPCs), and upon injury home in on to the sites of ischemia and initiate neovascularization, tissue/vessel remodeling, and cardiac regeneration most probably by paracrine effects only (Losordo and Dimmeler, 2004). Age-related and disease-linked impairment of EPC may contribute to incorrect vascularization and may be related to a loss of antioxidative defense. EPCs express antioxidative enzymes, like catalase, glutathione peroxidase-1, and manganese superoxide dismutase, which make them resistant to oxidative stress (Dernbach et al., 2004). Therefore, it has been proposed that genetic modifications of EPCs might restore and/or improve their therapeutic function. In fact, experimental overexpression of manganese superoxide dismutase ( $\mathrm{He}$ et al., 2004) enhanced EPC protection, whereas knockout of glutathione peroxidase- 1 gene diminished the viability and impaired vasculogenic potency of EPC (Galasso et al., 2006). Our study demonstrated that the same may apply to $\mathrm{HO} 1$ because the function of EPC in vitro 
and in vivo is impaired in mice that lack this gene (Deshane et al., 2007).

It has been shown that the introduction of rabbit EPCs into the denuded arteries enhanced the process of reendothelialization (Kong et al., 2004). Additionally, retroviral overexpression of endothelial nitric oxide synthase in those EPCs significantly improved endothelial regeneration. Transduction with human $H O 1$, however, did not affect the capacity of EPCs which, in the authors' opinion, was probably related to the insufficient HO1 levels to generate $\mathrm{CO}$ in amounts required to enhance endothelial cell proliferation (Kong et al., 2004). Further examination of engineered stem cells, EPCs and other tissue progenitors overexpressing $\mathrm{HO} 1$ would be of great interest. According to our latest data, HO1 may be a major regulator of microRNA transcriptome because it strongly regulates the expression of many individual microRNAs affecting satellite progenitor cells differentiation to skeletal muscle (Ciesla et al., 2010).

\section{Conclusions}

In this review, we discuss the functional significance of the $\mathrm{HO} 1$ system in the vascular bed and the potential therapeutic applications of $\mathrm{HO} 1$ gene transfer in cardiovascular disease. Available data in animal models indicate that patients suffering from cardiovascular pathologies might benefit from cardiovascular gene therapies based on antioxidative, anti-apoptotic, anti-inflammatory and proangiogenic properties of $\mathrm{HO} 1$ activity products, namely $\mathrm{CO}$ and biliverdin/bilirubin. Moreover, it has been proposed that moderate, tightly controlled $\mathrm{HO1}$ expression by gene therapy should provide protection against various toxic insults including reactive iron released in heme degradation. It is important to emphasize that the treatment of human monogenic disorders with gene therapy is now a reality and advances in scientific and medical technologies may eventually create a possibility for us to provide custom genetic therapy of cardiovascular disease.

\section{Acknowledgments}

This work was supported by grants N301 009639 and N301 314837 from the Ministry of Science and Higher Education. The Faculty of Biochemistry, Biophysics and Biotechnology of the Jagiellonian University is a beneficiary of the structural funds from the European Union (grant No: POIG. 02.01.00-12-064/08 - Molecular biotechnology for health and POIG 01.02-00-109/99 Innovative methods of stem cell appli- cations in medicine; POIG.02.02.00-014/08 - Jagiellonian Centre of Experimental Therapeutics; POIG.01.01.02-00069/09 - Vascular endothelium in civilization diseases. Alicja Jozkowicz was a recipient of the Wellcome Trust Senior Research Fellowship in Basic Biomedical Science.

\section{Abbreviations}

BVR, biliverdin reductase; EPCs, endothelial progenitor cells; HSE, heat shock element; HIF $1 \alpha$, hypoxia inducible factor $1 \alpha$; HO1, heme oxygenase 1; HRE, hypoxia response element; PHD, prolyl hydroxylase; ROS, reactive oxygen species; SDF1, stromal cell-derived factor 1 ; Tet, tetracycline; VEGF, vascular endothelial growth factor A.

\section{References}

Ahmad Z., Salim M., Maines M.D. (2002) Human biliverdin reductase is a leucine zipper-like DNA-binding protein and functions in transcriptional activation of heme oxygenase1 by oxidative stress. J. Biol. Chem. 277: 9226-9232.

Aiuti A., Cattaneo F., Galimberti S., Benninghoff U., Cassani B., Callegaro L., Scaramuzza S., Andolfi G., Mirolo M., Brigida I. et al. (2009) Gene therapy for immunodeficiency due to adenosine deaminase deficiency. N. Engl. J. Med. 360: 447-458.

Baranano D.E., Wolosker H., Bae B.I., Barrow R.K., Snyder S.H., Ferris C.D. (2000) A mammalian iron ATPase induced by iron. J. Biol. Chem. 275: 15166-15173.

Bhang S.H., Kim J.H., Yang H.S., La W.G., Lee T.J., Kim G.H., Kim H.A., Lee M., Kim B.S. (2010) Combined gene therapy with hypoxia-inducible factor-1alpha and heme oxygenase-1 for therapeutic angiogenesis. Tissue Eng. Part A. 17(7-8): 915-926.

Brydun A., Watari Y., Yamamoto Y., Okuhara K., Teragawa H., Kono F., Chayama K., Oshima T., Ozono R. (2007) Reduced expression of heme oxygenase-1 in patients with coronary atherosclerosis. Hypertens. Res. 30: 341-348.

Bussolati B., Ahmed A., Pemberton H., Landis R.C., Di Carlo F., Haskard D.O., Mason J.C. (2004) Bifunctional role for VEGF-induced heme oxygenase-1 in vivo: induction of angiogenesis and inhibition of leukocytic infiltration. Blood 103: 761-766.

Cartier N., Hacein-Bey-Abina S., Bartholomae C.C., Veres G., Schmidt M., Kutschera I., Vidaud M., Abel U., Dal-Cortivo L., Caccavelli L. et al. (2009) Hematopoietic stem cell gene therapy with a lentiviral vector in X-linked adrenoleukodystrophy. Science 326: 818-823.

Cavazzana-Calvo M., Hacein-Bey S., de Saint Basile G., Gross F., Yvon E., Nusbaum P., Selz F., Hue C., Certain S., Casanova J.L. et al. (2000) Gene therapy of human severe combined immunodeficiency (SCID)-X1 disease. Science 288: 669-672.

Cavazzana-Calvo M., Payen E., Negre O., Wang G., Hehir K., Fusil F., Down J., Denaro M., Brady T., Westerman K., 
et al. (2010) Transfusion independence and HMGA2 activation after gene therapy of human beta-thalassaemia. Nature 467: 318-322.

Ceradini D.J., Gurtner G.C. (2005) Homing to hypoxia: HIF-1 as a mediator of progenitor cell recruitment to injured tissue. Trends Cardiovasc. Med. 15: 57-63.

Chen Y.H., Lin S.J., Lin M.W., Tsai H.L., Kuo S.S., Chen J.W., Charng M.J., Wu T.C., Chen L.C., Ding Y.A. et al. (2002) Microsatellite polymorphism in promoter of heme oxygenase-1 gene is associated with susceptibility to coronary artery disease in type 2 diabetic patients. Hum. Genet. 111: 1-8.

Choi Y.K., Kim C.K., Lee H., Jeoung D., Ha K.S., Kwon Y.G., Kim K.W., Kim Y.M. (2010) Carbon monoxide promotes VEGF expression by increasing HIF-1alpha protein level via two distinct mechanisms, translational activation and stabilization of HIF-1alpha protein. J. Biol. Chem. 285: 32116-32125.

Ciesla M., Kozakowska M., Skrzypek K., Stefanska A., Loboda A., Jazwa A., Was H., Grochot-Przeczek A., Kotlinowski J., Dyduch G. et al. (2010) Role of mirnas in heme oxygenase-1 mediated impairment of myoblast differentiation. Circulation, Abstract 20095.

Coceani F. (1993) Carbon monoxide and dilation of blood vessels. Science 260: 739.

Coffman L.G., Parsonage D., D'Agostino R., Jr., Torti F.M., Torti S.V. (2009) Regulatory effects of ferritin on angiogenesis. Proc. Natl. Acad. Sci. U S A 106: 570-575.

Czibik G., Sagave J., Martinov V., Ishaq B., Sohl M., Sefland I., Carlsen H., Farnebo F., Blomhoff R., Valen G. (2009) Cardioprotection by hypoxia-inducible factor 1 alpha transfection in skeletal muscle is dependent on haem oxygenase activity in mice. Cardiovasc. Res. 82: 107-114.

Deramaudt B.M., Braunstein S., Remy P., Abraham N.G. (1998) Gene transfer of human heme oxygenase into coronary endothelial cells potentially promotes angiogenesis. J. Cell Biochem. 68: 121-127.

Dernbach E., Urbich C., Brandes R.P., Hofmann W.K., Zeiher A.M., Dimmeler S. (2004) Antioxidative stress-associated genes in circulating progenitor cells: evidence for enhanced resistance against oxidative stress. Blood 104: 3591-3597.

Deshane J., Chen S., Caballero S., Grochot-Przeczek A., Was H., Li Calzi S., Lach R., Hock T.D., Chen B., Hill-Kapturczak N. et al. (2007) Stromal cell-derived factor 1 promotes angiogenesis via a heme oxygenase 1-dependent mechanism. J. Exp. Med. 204: 605-618.

Dick P., Schillinger M., Minar E., Mlekusch W., Amighi J., Sabeti S., Schlager O., Raith M., Endler G., Mannhalter C. et al. (2005) Haem oxygenase-1 genotype and cardiovascular adverse events in patients with peripheral artery disease. Eur. J. Clin. Invest. 35: 731-737.

Dulak J., Jozkowicz A., Foresti R., Kasza A., Frick M., Huk I., Green C.J., Pachinger O., Weidinger F., Motterlini R. (2002) Heme oxygenase activity modulates vascular endothelial growth factor synthesis in vascular smooth muscle cells. Antioxid. Redox Signal 4: 229-240.
Dulak J., Jozkowicz A. (2003) Carbon monoxide - a "new" gaseous modulator of gene expression. Acta Biochim. Pol. 50: 31-47.

Dulak J., Deshane J., Jozkowicz A., Agarwal A. (2008) Heme oxygenase-1 and carbon monoxide in vascular pathobiology: focus on angiogenesis. Circulation 117: 231-241.

Eisenstein R.S., Garcia-Mayol D., Pettingell W., Munro H.N. (1991) Regulation of ferritin and heme oxygenase synthesis in rat fibroblasts by different forms of iron. Proc. Natl. Acad. Sci. U S A 88: 688-692.

Exner M., Minar E., Wagner O., Schillinger M. (2004) The role of heme oxygenase-1 promoter polymorphisms in human disease. Free Radic. Biol. Med. 37: 1097-1104.

Ferrara N. (1999) Role of vascular endothelial growth factor in the regulation of angiogenesis. Kidney Int. 56: 794-814.

Ferris C.D., Jaffrey S.R., Sawa A., Takahashi M., Brady S.D., Barrow R.K., Tysoe S.A., Wolosker H., Barańano D.E., Doré S. et al. (1999) Haem oxygenase-1 prevents cell death by regulating cellular iron. Nat. Cell Biol. 1: 152-157.

Florczyk U.M., Jozkowicz A., Dulak J. (2008) Biliverdin reductase: new features of an old enzyme and its potential therapeutic significance. Pharmacol. Rep. 60: 38-48.

Florczyk U., Golda S., Zieba A., Cisowski J., Jozkowicz A., Dulak J. (2010a) Overexpression of biliverdin reductase enhances resistance to chemotherapeutics. Cancer Lett. 300: 40-47.

Florczyk U., Loboda A., Stachurska A., Jozkowicz A., Dulak J. (2010b) Role of Nrf2 transcription factor in cellular response to oxidative stress. Post. Biochem. 56: 147-155.

Forstermann U., Munzel T. (2006) Endothelial nitric oxide synthase in vascular disease: from marvel to menace. Circulation 113: 1708-1714.

Funk M., Endler G., Schillinger M., Mustafa S., Hsieh K., Exner M., Lalouschek W., Mannhalter C., Wagner O. (2004) The effect of a promoter polymorphism in the heme oxygenase-1 gene on the risk of ischaemic cerebrovascular events: the influence of other vascular risk factors. Thromb. Res. 113: 217-223.

Galasso G., Schiekofer S., Sato K., Shibata R., Handy D.E., Ouchi N., Leopold J.A., Loscalzo J., Walsh K. (2006) Impaired angiogenesis in glutathione peroxidase-1-deficient mice is associated with endothelial progenitor cell dysfunction. Circ. Res. 98: 254-261.

Grochot-Przeczek A., Dulak J., Jozkowicz A. (2010) Heme oxygenase-1 in neovascularisation: A diabetic perspective. Thromb. Haemost. 104: 424-431.

Gulesserian T., Wenzel C., Endler G., Sunder-Plassmann R., Marsik C., Mannhalter C., Iordanova N., Gyongyosi M., Wojta J., Mustafa S. et al. (2005) Clinical restenosis after coronary stent implantation is associated with the heme oxygenase-1 gene promoter polymorphism and the heme oxygenase-1 +99G/C variant. Clin. Chem. 51: 1661-1665.

Hacein-Bey-Abina S., Le Deist F., Carlier F., Bouneaud C., Hue C., De Villartay J.P., Thrasher A.J., Wulffraat N., Sorensen R., Dupuis-Girod S. et al. (2002) Sustained cor- 
rection of X-linked severe combined immunodeficiency by ex vivo gene therapy. N. Engl. J. Med. 346: 1185-1193.

Hamamura R.S., Ohyashiki J.H., Kurashina R., Kobayashi C., Zhang Y., Takaku T., Ohyashiki K. (2007) Induction of heme oxygenase-1 by cobalt protoporphyrin enhances the antitumour effect of bortezomib in adult T-cell leukaemia cells. Br. J. Cancer 97: 1099-1105.

He T., Peterson T.E., Holmuhamedov E.L., Terzic A., Caplice N.M., Oberley L.W., Katusic Z.S. (2004) Human endothelial progenitor cells tolerate oxidative stress due to intrinsically high expression of manganese superoxide dismutase. Arterioscler. Thromb. Vasc. Biol. 24: 2021-2027.

Hill-Kapturczak N., Sikorski E., Voakes C., Garcia J., Nick H.S., Agarwal A. (2003) An internal enhancer regulates hemeand cadmium-mediated induction of human heme oxygenase-1. Am. J. Physiol. Renal. Physiol. 285: F515-523.

Hirai H., Kubo H., Yamaya M., Nakayama K., Numasaki M., Kobayashi S., Suzuki S., Shibahara S., Sasaki H. (2003) Microsatellite polymorphism in heme oxygenase-1 gene promoter is associated with susceptibility to oxidant-induced apoptosis in lymphoblastoid cell lines. Blood 102: 1619-1621.

Jazwa A., Loboda A., Golda S., Cisowski J., Szelag M., Zagorska A., Sroczynska P., Drukala J., Jozkowicz A., Dulak J. (2006) Effect of heme and heme oxygenase-1 on vascular endothelial growth factor synthesis and angiogenic potency of human keratinocytes. Free Radic. Biol. Med. 40: 1250-1263.

Jazwa A., Jozkowicz A., Dulak J. (2007) New vectors and strategies for cardiovascular gene therapy. Curr. Gene Ther. 7: 7-23.

Jazwa A., Cuadrado A. (2010) Targeting heme oxygenase-1 for neuroprotection and neuroinflammation in neurodegenerative diseases. Curr. Drug. Targets 11: 1517-1531.

Jozkowicz A., Dulak J. (2003a) Effects of protoporphyrins on production of nitric oxide and expression of vascular endothelial growth factor in vascular smooth muscle cells and macrophages. Acta Biochim. Pol. 50: 69-79.

Jozkowicz A., Huk I., Nigisch A., Weigel G., Dietrich W., Motterlini R., Dulak J. (2003b) Heme oxygenase and angiogenic activity of endothelial cells: stimulation by carbon monoxide and inhibition by tin protoporphyrin-IX. Antioxid. Redox. Signal 5: 155-162.

Juan S.H., Lee T.S., Tseng K.W., Liou J.Y., Shyue S.K., Wu K.K., Chau L.Y. (2001) Adenovirus-mediated heme oxygenase-1 gene transfer inhibits the development of atherosclerosis in apolipoprotein E-deficient mice. Circulation 104: 1519-1525.

Kitamuro T., Takahashi K., Ogawa K., Udono-Fujimori R., Takeda K., Furuyama K., Nakayama M., Sun J., Fujita H., Hida W. et al. (2003) Bach1 functions as a hypoxia-inducible repressor for the heme oxygenase-1 gene in human cells. J. Biol. Chem. 278: 9125-9133.

Kong D., Melo L.G., Mangi A.A., Zhang L., Lopez-Ilasaca M., Perrella M.A., Liew C.C., Pratt R.E., Dzau V.J. (2004) Enhanced inhibition of neointimal hyperplasia by genetically engineered endothelial progenitor cells. Circulation 109: 1769-1775.
Lakkisto P., Kyto V., Forsten H., Siren J.M., Segersvard H., Voipio-Pulkki L.M., Laine M., Pulkki K., Tikkanen I. (2010) Heme oxygenase-1 and carbon monoxide promote neovascularization after myocardial infarction by modulating the expression of HIF-1alpha, SDF-1alpha and VEGF-B. Eur. J. Pharmacol. 635: 156-164.

Lee P.J., Jiang B.H., Chin B.Y., Iyer N.V., Alam J., Semenza G.L., Choi A.M. (1997) Hypoxia-inducible factor-1 mediates transcriptional activation of the heme oxygenase-1 gene in response to hypoxia. J. Biol. Chem. 272: 5375-5381.

Li Volti G., Sacerdoti D., Sangras B., Vanella A., Mezentsev A., Scapagnini G., Falck J.R., Abraham N.G. (2005) Carbon monoxide signaling in promoting angiogenesis in human microvessel endothelial cells. Antioxid. Redox. Signal 7: 704-710.

Lin H.H., Chen Y.H., Chang P.F., Lee Y.T., Yet S.F., Chau L.Y. (2008) Heme oxygenase-1 promotes neovascularization in ischemic heart by coinduction of VEGF and SDF-1. J. Mol. Cell Cardiol. 45: 44-55.

Lin H.H., Chen Y.H., Yet S.F., Chau L.Y. (2009) After vascular injury, heme oxygenase-1/carbon monoxide enhances reendothelialization via promoting mobilization of circulating endothelial progenitor cells. J. Thromb Haemost. 7: 1401-1408.

Lin Q., Weis S., Yang G., Weng Y.H., Helston R., Rish K., Smith A., Bordner J., Polte T., Gaunitz F. et al. (2007) Heme oxygenase-1 protein localizes to the nucleus and activates transcription factors important in oxidative stress. J. Biol. Chem. 282: 20621-20633.

Loboda A., Jazwa A., Grochot-Przeczek A., Rutkowski A.J., Cisowski J., Agarwal A., Jozkowicz A., Dulak J. (2008) Heme oxygenase-1 and the vascular bed: from molecular mechanisms to therapeutic opportunities. Antioxid. Redox. Signal 10: 1767-1812.

Losordo D.W., Dimmeler S. (2004) Therapeutic angiogenesis and vasculogenesis for ischemic disease: part II: cellbased therapies. Circulation 109: 2692-2697.

Lublinghoff N., Winkler K., Winkelmann B.R., Seelhorst U., Wellnitz B., Boehm B.O., März W., Hoffmann M.M. (2009) Genetic variants of the promoter of the heme oxygenase-1 gene and their influence on cardiovascular disease (the Ludwigshafen Risk and Cardiovascular Health study). BMC Med. Genet. 10: 36.

Maguire A.M., High K.A., Auricchio A., Wright J.F., Pierce E.A., Testa F., Mingozzi F., Bennicelli J.L., Ying G.S., Rossi S. et al. (2009) Age-dependent effects of RPE65 gene therapy for Leber's congenital amaurosis: a phase 1 dose-escalation trial. Lancet 374: 1597-1605.

Maines M.D., Trakshel G.M., Kutty R.K. (1986) Characterization of two constitutive forms of rat liver microsomal heme oxygenase. Only one molecular species of the enzyme is inducible. J. Biol. Chem. 261: 411-419.

Maines M.D. (1988) Heme oxygenase: function, multiplicity, regulatory mechanisms, and clinical applications. FASEB J. 2: 2557-2568. 
Maines M.D. (1997) The heme oxygenase system: a regulator of second messenger gases. Ann. Rev. Pharmacol. Toxicol. 37: 517-554.

Melo L.G., Agrawal R., Zhang L., Rezvani M., Mangi A.A., Ehsan A., Griese D.P., Dell'Acqua G., Mann M.J., Oyama J. et al. (2002) Gene therapy strategy for long-term myocardial protection using adeno-associated virus-mediated delivery of heme oxygenase gene. Circulation 105: 602-607.

Miralem T., Hu Z., Torno M.D., Lelli K.M., Maines M.D. (2005) Small interference RNA-mediated gene silencing of human biliverdin reductase, but not that of heme oxygenase-1, attenuates arsenite-mediated induction of the oxygenase and increases apoptosis in 293A kidney cells. J. Biol. Chem. 280: 17084-17092.

Morgan L., Hawe E., Palmen J., Montgomery H., Humphries S.E., Kitchen N. (2005) Polymorphism of the heme oxygenase-1 gene and cerebral aneurysms. Br. J. Neurosurg. 19: 317-321.

Muller R.M., Taguchi H., Shibahara S. (1987) Nucleotide sequence and organization of the rat heme oxygenase gene. J. Biol. Chem. 262: 6795-6802.

Neufeld G., Cohen T., Gengrinovitch S., Poltorak Z. (1999) Vascular endothelial growth factor (VEGF) and its receptors. FASEB J. 13: 9-22.

Ogawa K., Sun J., Taketani S., Nakajima O., Nishitani C., Sassa S., Hayashi N., Yamamoto M., Shibahara S., Fujita H., et al. (2001) Heme mediates derepression of Maf recognition element through direct binding to transcription repressor Bach1. EMBO J. 20: 2835-2843.

Olivetti G., Quaini F., Sala R., Lagrasta C., Corradi D., Bonacina E., Gambert S.R., Cigola E., Anversa P. (1996) Acute myocardial infarction in humans is associated with activation of programmed myocyte cell death in the surviving portion of the heart. J. Mol. Cell. Cardiol. 28: 2005-2016.

Outomuro D., Grana D.R., Azzato F., Milei J. (2007) Adriamycin-induced myocardial toxicity: new solutions for an old problem? Int. J. Cardiol. 117: 6-15.

Oyake T., Itoh K., Motohashi H., Hayashi N., Hoshino H., Nishizawa M., Yamamoto M., Igarashi K. (1996) Bach proteins belong to a novel family of BTB-basic leucine zipper transcription factors that interact with MafK and regulate transcription through the NF-E2 site. Mol. Cell Biol. 16: 6083-6095.

Pachori A.S., Melo L.G., Hart M.L., Noiseux N., Zhang L., Morello F., Solomon S.D., Stahl G.L., Pratt R.E., Dzau V.J. (2004) Hypoxia-regulated therapeutic gene as a preemptive treatment strategy against ischemia/reperfusion tissue injury. Proc. Natl. Acad. Sci. USA 101: 12282-12287.

Pachori A.S., Melo L.G., Zhang L., Solomon S.D., Dzau V.J. (2006) Chronic recurrent myocardial ischemic injury is significantly attenuated by pre-emptive adeno-associated virus heme oxygenase-1 gene delivery. J. Am. Coll. Cardiol. 47: 635-643.

Ponka P. (1999) Cellular iron metabolism. Kidney Int. Suppl. 69: S2-11.
Poss K.D., Tonegawa S. (1997) Heme oxygenase 1 is required for mammalian iron reutilization. Proc. Natl. Acad. Sci. USA 94: 10919-10924.

Pouyssegur J., Dayan F., Mazure N.M. (2006) Hypoxia signalling in cancer and approaches to enforce tumour regression. Nature 441: 437-443.

Rissanen T.T., Yla-Herttuala S. (2007) Current status of cardiovascular gene therapy. Mol. Ther. 15: 1233-1247.

Roselli E.A., Mezzadra R., Frittoli M.C., Maruggi G., Biral E., Mavilio F., Mastropietro F., Amato A., Tonon G., Refaldi C. et al. (2010) Correction of beta-thalassemia major by gene transfer in haematopoietic progenitors of pediatric patients. EMBO Mol. Med. 2: 315-328.

Rotenberg M.O., Maines M.D. (1990) Isolation, characterization, and expression in Escherichia coli of a cDNA encoding rat heme oxygenase-2. J. Biol. Chem. 265: 7501-7506.

Ryter S.W., Alam J., Choi A.M. (2006) Heme oxygenase-1/carbon monoxide: from basic science to therapeutic applications. Physiol. Rev. 86: 583-650.

Sasayama S., Fujita M. (1992) Recent insights into coronary collateral circulation. Circulation 85: 1197-1204.

Semenza G.L. (2004) Hydroxylation of HIF-1: oxygen sensing at the molecular level. Physiology (Bethesda) 19: 176-182.

Slebos D.J., Ryter S.W., van der Toorn M., Liu F., Guo F., Baty C.J., Karlsson J.M., Watkins S.C., Kim H.P., Wang X., et al. (2007) Mitochondrial localization and function of heme oxygenase-1 in cigarette smoke-induced cell death. Am. J. Respir. Cell Mol. Biol. 36: 409-417.

Stachurska A., Florczyk U., Jozkowicz A., Dulak J., Loboda A. (2010) The new face of factors induced by hypoxia-HIF-1 and HIF-2 and oxidative stress. Post. Bioch. 56: 156-164.

Sun J., Hoshino H., Takaku K., Nakajima O., Muto A., Suzuki H., Tashiro S., Takahashi S., Shibahara S., Alam J. et al. (2002) Hemoprotein Bach1 regulates enhancer availability of heme oxygenase-1 gene. EMBO J. 21: 5216-5224.

Sunderkotter C., Steinbrink K., Goebeler M., Bhardwaj R., Sorg C. (1994) Macrophages and angiogenesis. J. Leukoc. Biol. 55: 410-422.

Suttner D.M., Dennery P.A. (1999) Reversal of HO-1 related cytoprotection with increased expression is due to reactive iron. FASEB J. 13: 1800-1809.

Suzuki H., Tashiro S., Hira S., Sun J., Yamazaki C., Zenke Y., Ikeda-Saito M., Yoshida M., Igarashi K. (2004) Heme regulates gene expression by triggering Crm1-dependent nuclear export of Bach1. EMBO J. 23: 2544-2553.

Suzuki M., Iso-o N., Takeshita S., Tsukamoto K., Mori I., Sato T., Ohno M., Nagai R., Ishizaka N. (2003) Facilitated angiogenesis induced by heme oxygenase-1 gene transfer in a rat model of hindlimb ischemia. Biochem. Biophys. Res. Commun. 302: 138-143.

Taha H., Skrzypek K., Guevara I., Nigisch A., Mustafa S., Grochot-Przeczek A., Ferdek P., Was H., Kotlinowski J., Kozakowska M. et al. (2010) Role of heme oxygenase-1 in human endothelial cells: lesson from the promoter allelic variants. Arterioscler. Thromb. Vasc. Biol. 30: 1634-1641. 
Takahashi S., Takahashi Y., Ito K., Nagano T., Shibahara S., Miura T. (1999) Positive and negative regulation of the human heme oxygenase-1 gene expression in cultured cells. Biochim. Biophys. Acta 1447: 231-235.

Tang Y.L., Tang Y., Zhang Y.C., Qian K., Shen L., Phillips M.I. (2004) Protection from ischemic heart injury by a vigilant heme oxygenase-1 plasmid system. Hypertension 43: 746751.

Tang Y.L., Qian K., Zhang Y.C., Shen L., Phillips M.I. (2005) $A$ vigilant, hypoxia-regulated heme oxygenase-1 gene vector in the heart limits cardiac injury after ischemia-reperfusion in vivo. J. Cardiovasc. Pharmacol. Ther. 10: 251-263.

Tenhunen R., Marver H.S., Schmid R. (1968) The enzymatic conversion of heme to bilirubin by microsomal heme oxygenase. Proc. Natl. Acad. Sci. USA 61: 748-755.

Tongers J., Knapp J.M., Korf M., Kempf T., Limbourg A., Limbourg F.P., Li Z., Fraccarollo D., Bauersachs J., Han X. et al. (2008) Haeme oxygenase promotes progenitor cell mobilization, neovascularization, and functional recovery after critical hindlimb ischaemia in mice. Cardiovasc. Res. 78: 294-300.

Torti F.M., Torti S.V. (2002) Regulation of ferritin genes and protein. Blood 99: 3505-3516.

Tulis D.A., Durante W., Liu X., Evans A.J., Peyton K.J., Schafer A.I. (2001) Adenovirus-mediated heme oxygenase1 gene delivery inhibits injury-induced vascular neointima formation. Circulation 104: 2710-2715.
Wang W., Kwok A.M., Chan J.Y. (2007) The p65 isoform of Nrf1 is a dominant negative inhibitor of ARE-mediated transcription. J. Biol. Chem. 282: 24670-24678.

Wiesener M.S., Jurgensen J.S., Rosenberger C., Scholze C.K., Horstrup J.H., Warnecke C., Mandriota S., Bechmann I., Frei U.A., Pugh C.W. et al. (2003) Widespread hypoxiainducible expression of HIF-2alpha in distinct cell populations of different organs. FASEB J. 17: 271-273.

Williams S.E., Wootton P., Mason H.S., Bould J., Iles D.E., Riccardi D., Peers C., Kemp P.J. (2004) Hemoxygenase-2 is an oxygen sensor for a calcium-sensitive potassium channel. Science 306: 2093-2097.

Yamada N., Yamaya M., Okinaga S., Nakayama K., Sekizawa K., Shibahara S., Sasaki H. (2000) Microsatellite polymorphism in the heme oxygenase-1 gene promoter is associated with susceptibility to emphysema. Am. J. Hum. Genet. 66: 187-195.

Yla-Herttuala S., Alitalo K. (2003) Gene transfer as a tool to induce therapeutic vascular growth. Nat. Med. 9: 694-701.

Yla-Herttuala S., Markkanen J.E., Rissanen T.T. (2004) Gene therapy for ischemic cardiovascular diseases: some lessons learned from the first clinical trials. Trends Cardiovasc. Med. 14: 295-300.

Yoshida T., Biro P., Cohen T., Muller R.M., Shibahara S. (1988) Human heme oxygenase $c D N A$ and induction of its mRNA by hemin. Eur. J. Biochem. 171: 457-461. 